\title{
A GENERAL ASYMPTOTIC FORMULA FOR PARTITION FUNCTIONS
}

\author{
NELSON A. BRIGHAM
}

1. Introduction. Practically all partition functions that have been considered have generating functions of the form

$$
g_{\gamma}(s)=\prod_{n=1}^{\infty} \frac{1}{\left(1-e^{-s n}\right)^{\gamma(n)}}=\sum_{k=0}^{\infty} a_{\gamma}(k) e^{-s k},
$$

where the real part of $s$ is positive and

$$
F_{\gamma}(x)=\sum_{n \leqq x} \gamma(n) \sim K x^{u} \log ^{v} x, \quad \gamma(n) \geqq 0, K>0, u>0 .
$$

Here $a_{\gamma}(0)=1$ and for $k$ positive

$$
a_{\gamma}(k)
$$

(3) $=\sum_{k=1 k_{1}+2 k_{2}+\cdots+l k l, k_{n} \neq 0} \prod_{n=1}^{l} \frac{\gamma(n)[\gamma(n)+1] \cdots\left\lceil\gamma(n)+k_{n}-1\right\rfloor}{k_{n} !}$.

Thus the partition $k=1 k_{1}+2 k_{2}+\cdots+l k_{l}$ of $k$ into $k_{1}$ ones, $k_{2}$ twos, and so on, is weighted in a certain way by the product shown, and various partition functions are obtained by choosing the function $\gamma(n)$ appropriately. For example, if $\gamma(n)=1$ for all $n$, then $a_{\gamma}(k)$ becomes the unrestricted partition function; if $\gamma(n)=n$ for all $n$, then $a_{\gamma}(k)$ becomes the plane partition of Wright [2];1 if $\gamma(n)=1$ for $r$ th powers and 0 otherwise, then $a_{\gamma}(k)$ is the number of partitions of $k$ into $r$ th powers; and so on.

The purpose of this paper is to establish the asymptotic formula

$$
\begin{aligned}
\log A_{\gamma}(k) \sim \frac{1}{u}\{K u \Gamma(u+2) \zeta(u+1)\}^{1 /(u+1)} \\
\cdot\{u+1\}^{(u-v) /(u+1)} k^{u /(u+1)}\{\log k\}^{v /(u+1)},
\end{aligned}
$$

where

$$
A_{\gamma}(k)=\sum_{n=0}^{k} a_{\gamma}(n) .
$$

We prove (4) by a method used by Hardy and Ramanujan [1] to obtain some special cases thereof, namely, we first prove that for $s$

Received by the editors December 8, 1948.

1 Numbers in brackets refer to the references at the end of the paper. 
tending to zero through positive values

$$
\log g_{\gamma}(s) \sim K\left(\frac{1}{s}\right)^{u}\left(\log \frac{1}{s}\right)^{\circ} \Gamma(u+1) \zeta(u+1),
$$

and then apply a Tauberian theorem proved by Hardy and Ramanujan in their paper.

If $a_{\gamma}(k)$ is nondecreasing with increasing $k$ from a certain point on,

$$
\frac{A_{\gamma}(k)+O(1)}{. k} \leqq a_{\gamma}(k) \leqq A_{\gamma}(k)
$$

and hence (4) is true for $\log a_{\gamma}(k)$. This is the case, for example, if $\gamma(1) \geqq 1$. Formula (4) is obviously true for $a_{\gamma}(k)$ under wider conditions than monotonicity, but on the other hand it is not always true for $a_{\gamma}(k)$; for example, it is obviously false if $\gamma(n)$ is 1 for even $n$ and 0 for odd $n$.

Furthermore:2 Suppose that $\gamma(n) \geqq 1$ whenever it is nonzero. Suppose that the set $S$ of positive integers for which $\gamma(n) \neq 0$ has the property that all positive integers not less than $c$ can be partitioned into integers from $S$. Then (4) is also true for $\log a_{\gamma}(k)$.

To prove this observe that $a_{\gamma}(k) \geqq a_{\gamma}(m)$ for $k-c \geqq m \geqq 1$. Hence

$$
A_{\gamma}(k-c) /(k-c) \leqq a_{\gamma}(k) \leqq A_{\gamma}(k) \quad \text { for } k>c
$$

and the conclusion follows from (4).

As a result of this, one can deduce asymptotic formulas for the logarithm of any unweighted partition function, provided only that the set of integers used is such that all sufficiently large integers can be partitioned into integers from the set. In particular, this is certainly true if the set of positive integers contains two coprime integers.

An important lacuna in the literature on partitions is the absence of an exact or asymptotic formula for the number $T(k)$ of partitions of $k$ into primes. From (4) and the prime number theorem we get only

$$
\log T(k) \sim \frac{2 \pi}{3^{1 / 2}}\left(\frac{k}{\log k}\right)^{1 / 2}
$$

which is given by Hardy and Ramanujan (formula 5.281 of [1]).

In the paper following this one we consider a weighted partition function $a(k)$ involving primes, namely that obtained by choosing $\gamma(n)$ in (1) as the $\Lambda(n)$ of prime number theory. We obtain there an

2 The author is indebted to the referee for this observation, which materially extends the scope of the paper. 
asymptotic formula for $A(k)=\sum_{n=0}^{k} a(n)$ under the assumption of the Riemann hypothesis. From (4) follows the weaker result

$$
\log A(k) \sim \pi(2 k / 3)^{1 / 2}
$$

without any unproved hypothesis. Since proof of monotonicity of $a(k)$ would seem to be extremely difficult (if true), we cannot assert (9) for $\log a(k)$. Note that (9) is the same as the asymptotic formula for $\log p(k)$, where $p(k)$ is the number of unrestricted partitions of $k$. The correspondence follows from the fact that

$$
\sum_{n \leq x} \Lambda(n) \sim x \sim \sum_{n \leq x} 1
$$

Since also

$$
\sum_{p \leq x} \log p \sim x,
$$

the powers of the primes may be disregarded in (9).

Besides (8) and (9) we give some further applications of (4) at the end of the paper.

2. Proof. In the following $s$ is real and positive. Both " $o$ " and " $O$ " refer to $s$ tending to zero.

From (1) we obtain

$$
\log g_{\gamma}(s)=\sum_{n=1}^{\infty} \gamma(n) \log \frac{1}{1-e^{-o n}}=\sum_{m=1}^{\infty} \frac{1}{m} \phi(m s)
$$

where

$$
\phi(s)=\sum_{n=1}^{\infty} \gamma(n) e^{-s n}
$$

We find an asymptotic formula for $\phi(s)$ for $s$ tending to zero, and use it to find an asymptotic formula for $\log g_{\gamma}(s)$. This is the previously mentioned technique of Hardy and Ramanujan [1, pp. 245261].

$$
\begin{aligned}
\phi(s)= & \gamma(1) e^{-\bullet}+\gamma(2) e^{-20}+\gamma(3) e^{-3 v}+\gamma(4) e^{-40}+\cdots \\
= & -\gamma(1)\left\{e^{-2 v}-e^{-\bullet}\right\}-\{\gamma(1)+\gamma(2)\}\left\{e^{-3 s}-e^{-20}\right\} \\
& -\{\gamma(1)+\gamma(2)+\gamma(3)\}\left\{e^{-4 v}-e^{-8 \bullet}\right\}-\cdots \\
= & -\sum_{a=1}^{\infty} \sum_{n=1}^{q} \gamma(n) \int_{a}^{q+1} \frac{d\left(e^{-v y}\right)}{d y} d y
\end{aligned}
$$




$$
\begin{aligned}
& =\sum_{q=1}^{\infty} \int_{q}^{q+1} F_{\gamma}(y) s e^{-s y} d y \\
& =\int_{1}^{\infty} F_{\gamma}(y) s e^{-\imath y} d y=\int_{0}^{\infty} F_{\gamma}\left(\frac{x}{s}\right) e^{-x} d x .
\end{aligned}
$$

Thus

$$
\phi(s)=\int_{0}^{\rho^{1 / 2}} F_{\gamma}\left(\frac{x}{s}\right) e^{-x} d x+\int_{s^{1 / 2}}^{\infty} F_{\gamma}\left(\frac{x}{s}\right) e^{-x} d x .
$$

$F_{\gamma}(x)$ is an increasing function, hence in the first integral of (10),

$$
F_{\gamma}\left(\frac{x}{s}\right) \leqq F_{\gamma}\left(\frac{1}{s^{1 / 2}}\right) \leqq C s^{-u / 2} \log ^{*} \frac{1}{s}, \quad \text { for } s<\frac{1}{e},
$$

and so

$$
\int_{s}^{0^{1 / 2}} F_{\gamma}\left(\frac{x}{s}\right) e^{-x} d x=O\left\{s^{(1-u) / 2} \log ^{\nu} \frac{1}{s}\right\}, \quad \text { for } s \text { tending to zero. }
$$

$s$ may be taken so small that, in the second integral of (10),

$$
F_{\gamma}\left(\frac{x}{s}\right)=K\left(\frac{x}{s}\right)^{u}\left(\log \frac{x}{s}\right)^{v}\left(1+\rho_{1}(x)\right)
$$

where $\left|\rho_{1}(x)\right|<\epsilon$ uniformly in $x, x \geqq s^{1 / 2}$. Then:

$$
\begin{aligned}
\phi(s)= & O\left\{s^{(1-u) / 2} \log ^{v} \frac{1}{s}\right\} \\
& +(1+o(1)) K \int_{a^{1 / 2}}^{\infty}\left(\frac{x}{s}\right)^{u}\left(\log \frac{x}{s}\right)^{0} e^{-x} d x .
\end{aligned}
$$

Put

$$
\Phi(s)=\int_{s^{1 / 2}}^{\infty}\left(\frac{x}{s}\right)^{u}\left(\log \frac{x}{s}\right)^{0} e^{-x} d x=\int_{s^{1 / 2}}^{1 / \rho^{1 / 2}}+\int_{1 / s^{1 / 2}}^{\infty}=\Phi_{1}(s)+\Phi_{2}(s) .
$$

For $\Phi_{1}(s),-1 / 2 \leqq \log x / \log (1 / s) \leqq 1 / 2$ and

$$
\begin{aligned}
\log ^{v} \frac{x}{s} & =\left(\log ^{v} \frac{1}{s}\right)\left(1+\frac{\log x}{\log (1 / s)}\right)^{v} \\
& =\left(\log ^{v} \frac{1}{s}\right)\left(1+\frac{\log x}{\log (1 / s)} \rho_{2}(x)\right)
\end{aligned}
$$

where $\left|\rho_{2}(x)\right| \leqq C_{v}$. Therefore, 


$$
\begin{aligned}
\Phi_{1}(s)= & \left(\frac{1}{s}\right)^{u}\left(\log \frac{1}{s}\right)^{v} \int_{s^{1 / 2}}^{1 / \rho^{1 / 2}} x^{u}\left(1+\frac{\log x}{\log (1 / s)} \rho_{2}(x)\right) e^{-x} d x \\
= & \left(\frac{1}{s}\right)^{u}\left(\log \frac{1}{s}\right)^{v} \int_{0}^{\infty} x^{u} e^{-x} d x-\left(\frac{1}{s}\right)^{u}\left(\log \frac{1}{s}\right)^{v} \int_{0}^{e^{1 / 2}} x^{u} e^{-x} d x \\
& -\left(\frac{1}{s}\right)^{u}\left(\log \frac{1}{s}\right)^{v} \int_{1 / s^{1 / 2}}^{\infty} x^{u} e^{-x} d x \\
& +\left(\frac{1}{s}\right)^{u}\left(\log \frac{1}{s}\right)^{v-1} \int_{s^{1 / 2}}^{1 / s^{1 / 2}} x^{u}(\log x) \rho_{2}(x) e^{-x} d x .
\end{aligned}
$$

We find:

$$
\begin{aligned}
\Phi_{1}(s)= & \left(\frac{1}{s}\right)^{u}\left(\log \frac{1}{s}\right)^{v} \Gamma(u+1)+o\left\{s^{(1-u) / 2} \log ^{v} \frac{1}{s}\right\} \\
& +o(1)+o\left\{\left(\frac{1}{s}\right)^{u}\left(\log \frac{1}{s}\right)^{v-1}\right\} .
\end{aligned}
$$

Also, $\Phi_{2}(s)=o(1)$.

Substituting $\Phi(s)=\Phi_{1}(s)+\Phi_{2}(s)$ into (11), we have

$$
\begin{aligned}
\phi(s)= & o\left\{s^{(1-u) / 2} \log ^{\star} \frac{1}{s}\right\} \\
& +\{1+o(1)\}\left\{K\left(\frac{1}{s}\right)^{u}\left(\log \frac{1}{s}\right)^{\circ} \Gamma(u+1)\right\} \\
& +o\left\{s^{(1-u) / 2} \log ^{\bullet} \frac{1}{s}\right\}+o(1) \\
& +o\left\{\left(\frac{1}{s}\right)^{u}\left(\log \frac{1}{s}\right)^{v-1}\right\}+o(1) .
\end{aligned}
$$

Hence

$$
\phi(s) \sim K\left(\frac{1}{s}\right)^{u}\left(\log \frac{1}{s}\right)^{\circ} \Gamma(u+1) .
$$

Now

$$
\begin{aligned}
\log g_{\gamma}(s) & =\sum_{m=1}^{\infty} \frac{1}{m} \phi(m s)=\sum_{1 \leq m \leq M}+\sum_{M<m<1 / s^{1 / 2}}+\sum_{1 / s / 1 / s m \leq 1 / s}+\sum_{m>1 / \bullet} \\
& =\phi_{1}(s)+\phi_{2}(s)+\phi_{3}(s)+\phi_{1}(s),
\end{aligned}
$$

where $M$ is such that $\sum_{m>M} 1 / m^{\psi+1}<\epsilon(M(\epsilon)$ is fixed). 
First:

$$
\begin{aligned}
\phi_{1}(s) & =\sum_{m=1}^{M} \frac{1}{m} \phi(m s) \\
& =\sum_{m=1}^{M} \frac{1}{m}\{1+o(1)\}\left\{K\left(\frac{1}{m s}\right)^{u}\left(\log \frac{1}{m s}\right)^{v} \Gamma(u+1)\right\} .
\end{aligned}
$$

But $\log m / \log (1 / s)<1 / 2$ for $\phi_{1}(s)$ and

$$
\begin{aligned}
&\left(\log \frac{1}{m s}\right)^{v}=\left(\log \frac{1}{s}\right)^{v}\left(1-\frac{\log m}{\log (1 / s)}\right)^{v} \\
&=\left(\log \frac{1}{s}\right)^{v}\left(1+\frac{\log m}{\log (1 / s)} R_{v}(m)\right) \\
& \text { where }\left|R_{v}(m)\right|<C_{v} .
\end{aligned}
$$

Thus

$$
\begin{aligned}
\phi_{1}(s) & =K\left(\frac{1}{s}\right)^{u}\left(\log \frac{1}{s}\right)^{v} \Gamma(u+1) \sum_{m=1}^{M} \frac{1+o(1)}{m^{u+1}} \\
& +K\left(\frac{1}{s}\right)^{u}\left(\log \frac{1}{s}\right)^{v-1} \Gamma(u+1) \sum_{m=1}^{M} \frac{R_{v}(m) \log m}{m^{u+1}}\{1+o(1)\} .
\end{aligned}
$$

But $M$ is fixed, and so

$$
\begin{aligned}
\phi_{1}(s)=K\left(\frac{1}{s}\right)^{u}\left(\log \frac{1}{s}\right)^{v} \Gamma(u+1) \zeta(u+1)\{1+o(1)\} \\
+o\left\{\epsilon\left(\frac{1}{s}\right)^{u}\left(\log \frac{1}{s}\right)^{v}\right\}+o\left\{\left(\frac{1}{s}\right)^{u}\left(\log \frac{1}{s}\right)^{v-1}\right\} .
\end{aligned}
$$

Secondly, for $\phi_{2}(s)$ we have

$$
\phi(m s)<C_{u v}\left(\frac{1}{m s}\right)^{u}\left(\log \frac{1}{m s}\right)^{v},
$$

and since $M<m<1 / s^{1 / 2}$

$$
\left(\log \frac{1}{m s}\right)^{v} \leqq C_{v}\left(\log \frac{1}{s}\right)^{v} \quad \text { for all } v .
$$

So,

$$
\phi_{2}(s)=O\left\{\epsilon\left(\frac{1}{s}\right)^{u}\left(\log \frac{1}{s}\right)^{v}\right\}
$$


Thirdly, for $\phi_{3}(s)$ we observe from the original definition that $\phi\left(m_{1} s\right)<\phi\left(m_{2} s\right)$ if $m_{1}>m_{2}$. Hence we may use for all $m$ the estimation for the smallest $m$, namely $m=1 / s^{1 / 2}$, and find

$$
\phi_{3}(s)<C_{u v}\left(\frac{1}{s}\right)^{u / 2}\left(\log \frac{1}{s}\right)^{v} \sum_{1}^{1 / \bullet} \frac{1}{m}
$$

or,

$$
\phi_{8}(s)=O\left\{\left(\frac{1}{s}\right)^{u / 2}\left(\log \frac{1}{s}\right)^{v+1}\right\} .
$$

Finally, in $\phi_{4}(s), m s>1$, and the definition of $\phi(m s)$ readily yields $\phi_{4}(s)=O(1)$. See (3.544) of Hardy-Ramanujan.

This gives

$$
\begin{aligned}
\log g_{\gamma}(s)=K( & \left.\frac{1}{s}\right)^{u}\left(\log \frac{1}{s}\right)^{v} \Gamma(u+1) \zeta(u+1)\{1+o(1)\} \\
+ & o\left\{\epsilon\left(\frac{1}{s}\right)^{u}\left(\log \frac{1}{s}\right)^{v}\right\} \\
+ & o\left\{\left(\frac{1}{s}\right)^{u}\left(\log \frac{1}{s}\right)^{v-1}\right\} \\
+ & O\left\{\epsilon\left(\frac{1}{s}\right)^{u}\left(\log \frac{1}{s}\right)^{v}\right\} \\
+ & O\left\{\left(\frac{1}{s}\right)^{u / 2}\left(\log \frac{1}{s}\right)^{v+1}\right\} \\
+ & O\{1\},
\end{aligned}
$$

and we have

$$
\log g_{\gamma}(s) \sim K\left(\frac{1}{s}\right)^{u}\left(\log \frac{1}{s}\right)^{v} \Gamma(u+1) \zeta(u+1) .
$$

Now we quote, for reference, Hardy and Ramanujan's Theorem A $[1$, p. 252]:

"Suppose that

$$
\lambda_{1} \geqq 0, \lambda_{n}>\lambda_{n-1}, \lambda_{n} \rightarrow \infty ; \frac{\lambda_{n}}{\lambda_{n-1}} \rightarrow 1 ; a_{n} \geqq 0, A>0, \alpha>0 ;
$$

$\sum a_{n} e^{-\lambda_{n}}$ is convergent for $s>0$; 


$$
f(s)=\sum a_{n} e^{-\lambda_{n} \theta}=\exp \left[\{1+o(1)\} A s^{-\alpha}\left\{\log \frac{1}{s}\right\}^{-\beta}\right]
$$

when $s \rightarrow 0$. Then

$$
A_{n}=a_{1}+\cdots+a_{n}=\exp \left[\{1+o(1)\} B \lambda_{n}^{\alpha /(1+\alpha)}\left(\log \lambda_{n}\right)^{-\beta /(1+\alpha)}\right]
$$

where $B=A^{1 /(1+\alpha)} \alpha^{-\alpha /(1+\alpha)}(1+\alpha)^{1+[\beta /(1+\alpha)]}$ when $n \rightarrow \infty$."

Applying this theorem with:

$$
\begin{aligned}
f(s) & =g_{\gamma}(s), & & A=K \Gamma(u+1) \zeta(u+1), \\
\lambda_{n} & =n=k, & \beta & =-v, \\
\alpha & =u, & &
\end{aligned}
$$

we obtain (4) at once from (6).

3. Further applications. A few applications were indicated in the introduction.

As a matter of interest we give some further applications.

A fairly general type of partition is obtained by using only components of the form:

$$
n=a_{0}+a_{1} m+a_{2} m^{2}+\cdots+a_{r} m^{r}, \quad a_{r}>0, r \geqq 1,
$$

where the $a$ 's are such as to give positive integral $n$ for positive integral $m$. For simplicity we also assume the polynomial to yield $n_{1}>n_{2}$ for $m_{1}>m_{2}$. The $m$ 's themselves may be restricted in various ways.

Let $m_{1}$ be the largest integer which gives $n \leqq x$ in (12). Then

$$
\begin{aligned}
a_{0}+\cdots+a_{r-1} m_{1}^{r-1} & +a_{r} m_{1}^{r} \\
& \leqq x<a_{0}+\cdots+a_{r-1}\left(m_{1}+1\right)^{r-1}+a_{r}\left(m_{1}+1\right)^{r} .
\end{aligned}
$$

As $x \rightarrow \infty, m_{1} \rightarrow \infty$, and so for $x$ tending to infinity we have

$$
\begin{gathered}
x=a_{r} m_{1}^{r}+O\left(m_{1}^{r-1}\right)=a_{r} m_{1}^{r}\left(1+O\left(\frac{1}{m_{1}}\right)\right)=a_{r} m_{1}^{r}(1+o(1)), \\
m_{1}=\left(\frac{x}{a_{r}}\right)^{1 / r}(1+o(1)) .
\end{gathered}
$$

Let $m$ be restricted in some manner such that

$$
\sum_{m \leqq m_{1}} 1 \sim \kappa m_{1}^{\mu}\left(\log m_{1}\right)^{\nu}, \quad \mu>0 .
$$

Obviously, $\mu \leqq 1$. But when only the restricted $m$ 's of (13) are used in (12), $\sum_{m \leqq m_{1}} 1=\sum_{n \leqq x} 1$. Thus: 


$$
\sum_{n \leqq x} 1=\frac{\kappa}{r^{\nu}}\left(\frac{x}{a_{r}}\right)^{\mu / r}(\log x) \cdot\{1+o(1)\} .
$$

We put $\gamma(n)=1$ when $n$ is of the form (12), and $\gamma(n)=0$ otherwise. Comparing (14) with (2) and (4), we have the theorem:

If $A_{\gamma}(k)$ is the summatory function for the number of partitions of $k$ into components of the form

$n=a_{0}+a_{1} m+a_{2} m^{2}+\cdots+a_{r} m^{r}, \quad a_{r}>0, r \geqq 1, n>0, m>0$, $n_{1}>n_{2}$ for $m_{1}>m_{2}$,

where $\sum_{m \leqq m_{1}} 1 \sim \kappa m_{1}^{\mu}\left(\log m_{1}\right)^{\nu}, \mu>0$, then

$\log A_{\gamma}(k) \sim \mu^{-\mu /(r+\mu)}(r+\mu)^{(\mu-r \nu) /(r+\mu)}$

$$
\cdot\left\{\kappa \Gamma\left(\frac{\mu}{r}+2\right) \zeta\left(\frac{\mu}{r}+1\right)\right\}^{r /(r+\mu)}\left\{\frac{k}{a_{r}}\right\}^{\mu /(r+\mu)}\{\log k\}^{r \nu /(r+\mu)} .
$$

We apply this theorem to the following cases:

(A) Let $m$ be unrestricted. Then $\kappa=1, \mu=1, \nu=0$ and

$\log A_{r}(k) \sim(r+1)^{1 /(r+1)}\left\{\Gamma\left(\frac{1}{r}+2\right) \zeta\left(\frac{1}{r}+1\right)\right\}^{r /(r+1)}\left\{\frac{k}{a_{r}}\right\}^{1 /(r+1)}$.

Hardy and Ramanujan's result for partitions into $r$ th powers of positive integers $[1$, p. 259], is a special case of this. (Wright [3] gives more detailed results.)

(B) Let $m$ be restricted to primes. Then $\kappa=1, \mu=1, \nu=-1$ and $\log A_{r}(k) \sim(r+1)\left\{\Gamma\left(\frac{1}{r}+2\right) \zeta\left(\frac{1}{r}+1\right)\right\}^{r /(r+1)}$

$$
\cdot\left\{\frac{k}{a_{r}}\right\}^{1 /(r+1)}\{\log k\}^{-r /(r+1)} .
$$

Again, Hardy and Ramanujan's result for partitions into $r$ th powers of primes $[1$, p. 260] is included as a special case.

(C) Let $m$ be square-free. Then $\sum_{m \leqq m_{1}} 1 \sim 6 m_{1} / \pi^{2}$ and $\log A_{\gamma}(k)$

$$
\sim(r+1)^{1 /(r+1)}\left\{\frac{6}{\pi^{2}} \Gamma\left(\frac{1}{r}+2\right) \zeta\left(\frac{1}{r}+1\right)\right\}^{r /(r+1)}\left\{\frac{k}{a_{r}}\right\}^{1 /(r+1)} .
$$

In particular, for partitions into square-free numbers:

$$
\log a_{\gamma}(k) \sim 2 k^{1 / 2} \text {. }
$$


(D) Let $m$ be a polygonal number of order $q+2(q \geqq 1)$ :

$$
m=l+q\left(l^{2}-l\right) / 2 ; \quad l=1,2,3, \cdots
$$

Then

$$
\sum_{m \leq m_{1}} 1 \sim\left(\frac{2 m_{1}}{q}\right)^{1 / 2}
$$

and

$\log A_{\gamma}(k) \sim(2 r+1)^{1 /(2 r+1)}$

$$
\cdot\left\{\left(\frac{2}{q}\right)^{1 / 2} \Gamma\left(\frac{1}{2 r}+2\right) \zeta\left(\frac{1}{2 r}+1\right)\right\}^{2 r /(2 r+1)}\left\{\frac{k}{a_{r}}\right\}^{1 /(2 r+1)} .
$$

In particular, for partitions into triangular numbers:

$$
\log a_{\gamma}(k) \sim\left\{\frac{3}{4}(6 \pi)^{1 / 2} \zeta\left(\frac{3}{2}\right)\right\}^{2 / 3} k^{1 / 3} .
$$

(E) Various combinations may be taken, with the answer of higher order dominating. For instance, partitions into square-free numbers and triangular numbers, with duplications omitted or not, as desired, gives the result:

$$
\log a_{\gamma}(k) \sim 2 k^{1 / 2} .
$$

\section{REFERENCES}

1. G. H. Hardy and S. Ramanujan, Asymptotic formulae for the distribution of integers of various types, Collected Papers of Srinivasa Ramanujan. Also in Proc. London Math. Soc. vol. 16 (1917) pp. 112-132.

2. E. M. Wright, Asymptotic partition formulae I. Plane partitions, Quart. J. Math. Oxford Ser. vol. 2 (1931) pp. 177-189.

3. - Asymptotic partition formulae III. Partitions into kth powers. Acta Math. vol. 63 (1934) pp. 143-191.

University of Pennsylvania 